\title{
ZnO/Cellulose Nanofibre Composites for Sustainable Sunlight-Driven Dye Degradation
}

Mostafa Dehghani ${ }^{1}$, Humayun Nadeem ${ }^{1}$, Vikram Singh Raghuwanshi ${ }^{1}$, Hamidreza Mahdavi ${ }^{2}$, Mark M. Banaszak Holl ${ }^{1}$, Warren Batchelor ${ }^{1, *}$

${ }^{1}$ Bioresource Processing Research Institute of Australia, Department of Chemical Engineering, Monash University, Clayton, VIC 3800, Australia

${ }^{2}$ Department of Chemical Engineering, Monash University, Clayton, VIC 3800, Australia.

*Corresponding Author, E-mail: Warren.Batchelor@monash.edu

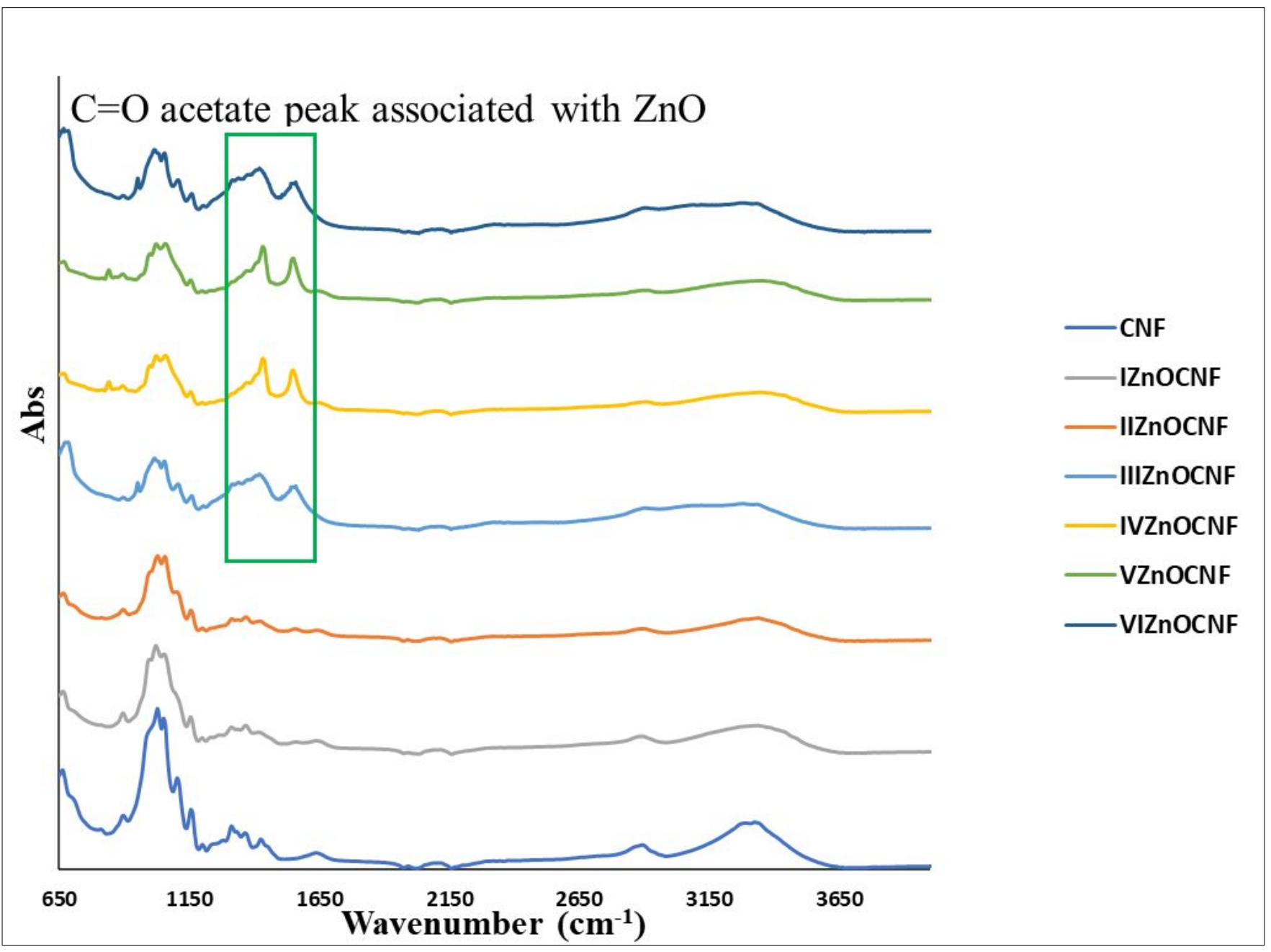

Fig. S1. FTIR spectra as a function of $\mathrm{ZnO}$ loading for the in-situ prepared $\mathrm{ZnO} / \mathrm{CNF}$ composites. 
Table S1. Pore size and surface area for all prepared samples using BET analysis.

\begin{tabular}{|c|c|c|}
\hline Data-set & Pore size $(\mathrm{nm})$ & Surface $\operatorname{area}\left(\mathrm{m}^{2} / \mathrm{g}\right)$ \\
\hline CNF & 249 & 0.11 \\
\hline ZnO & 23 & 12.15 \\
\hline IZnOCNF & 63 & 2.24 \\
\hline IIZnOCNF & 6.9 & 10.09 \\
\hline IIIZnOCNF & 6.4 & 11.91 \\
\hline IVZnOCNF & 3.1 & 12.06 \\
\hline VZnOCNF & 3.2 & 11.78 \\
\hline VIZnOCNF & 7.9 & 10.94 \\
\hline
\end{tabular}




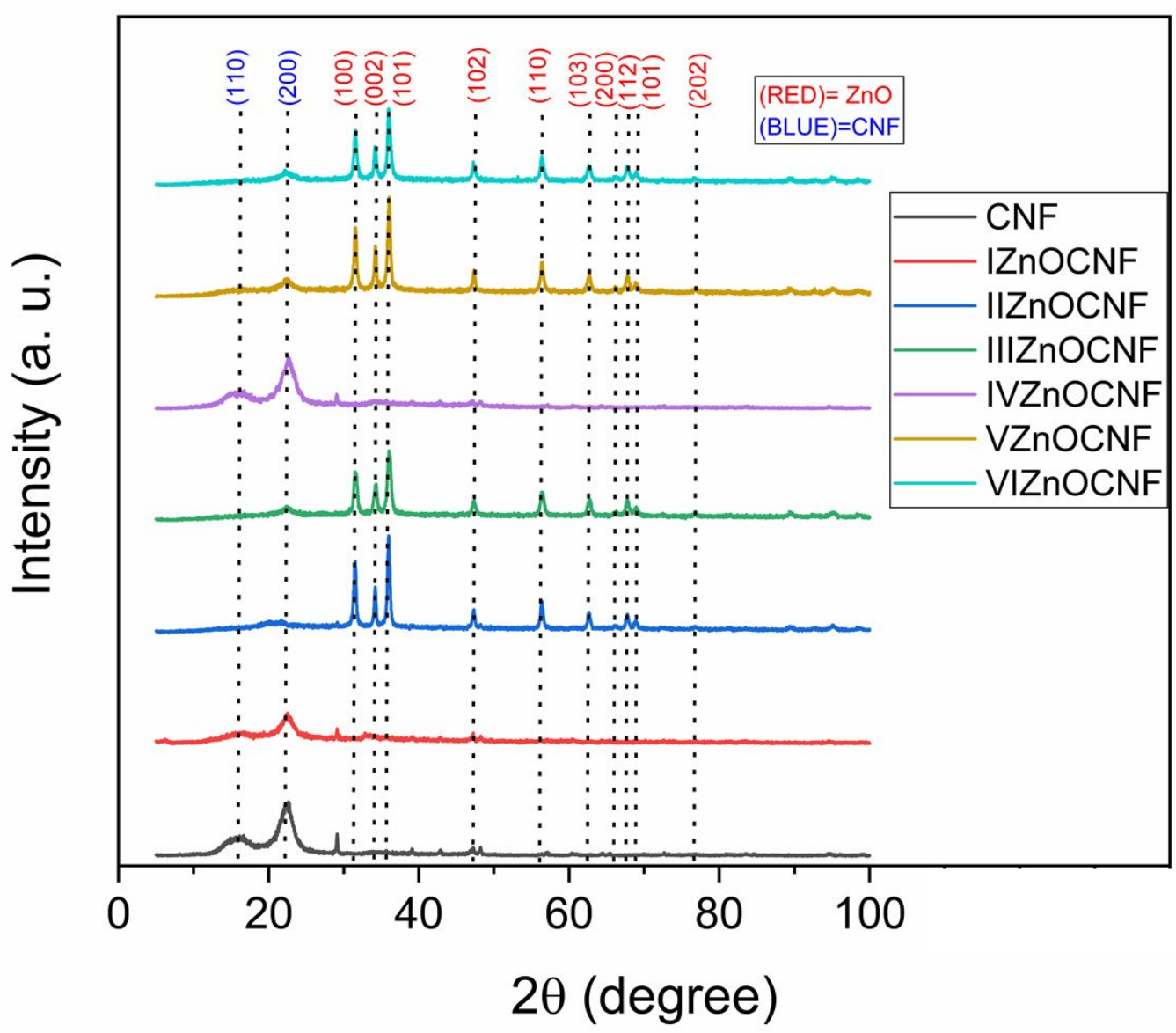

Fig. S2. a) XRD patterns of CNF and all synthesized catalysts. Annotations with blue color belong to $\mathrm{CNF}$ and with red color belong to $\mathrm{ZnO}$. 
a)
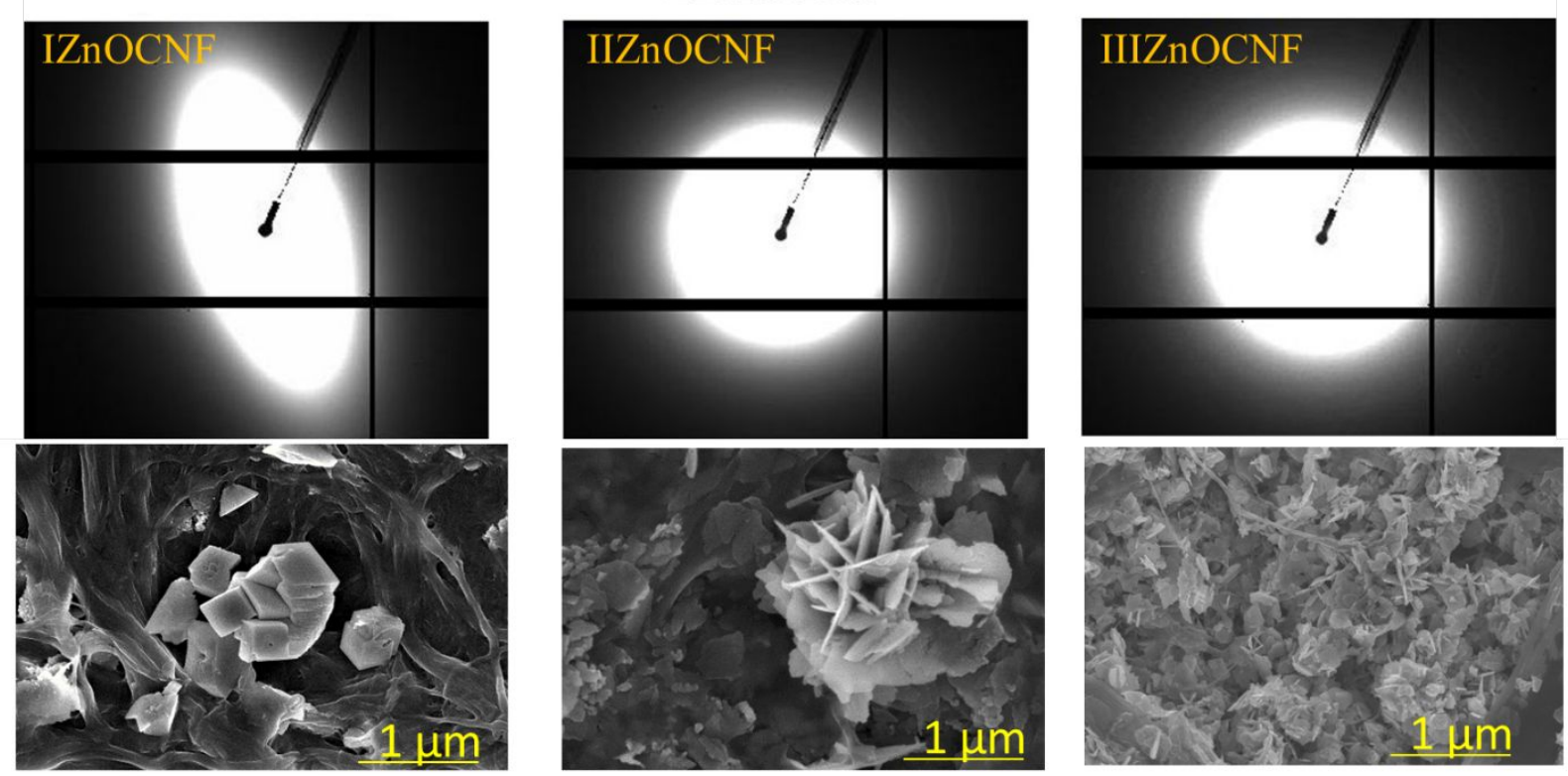

b)

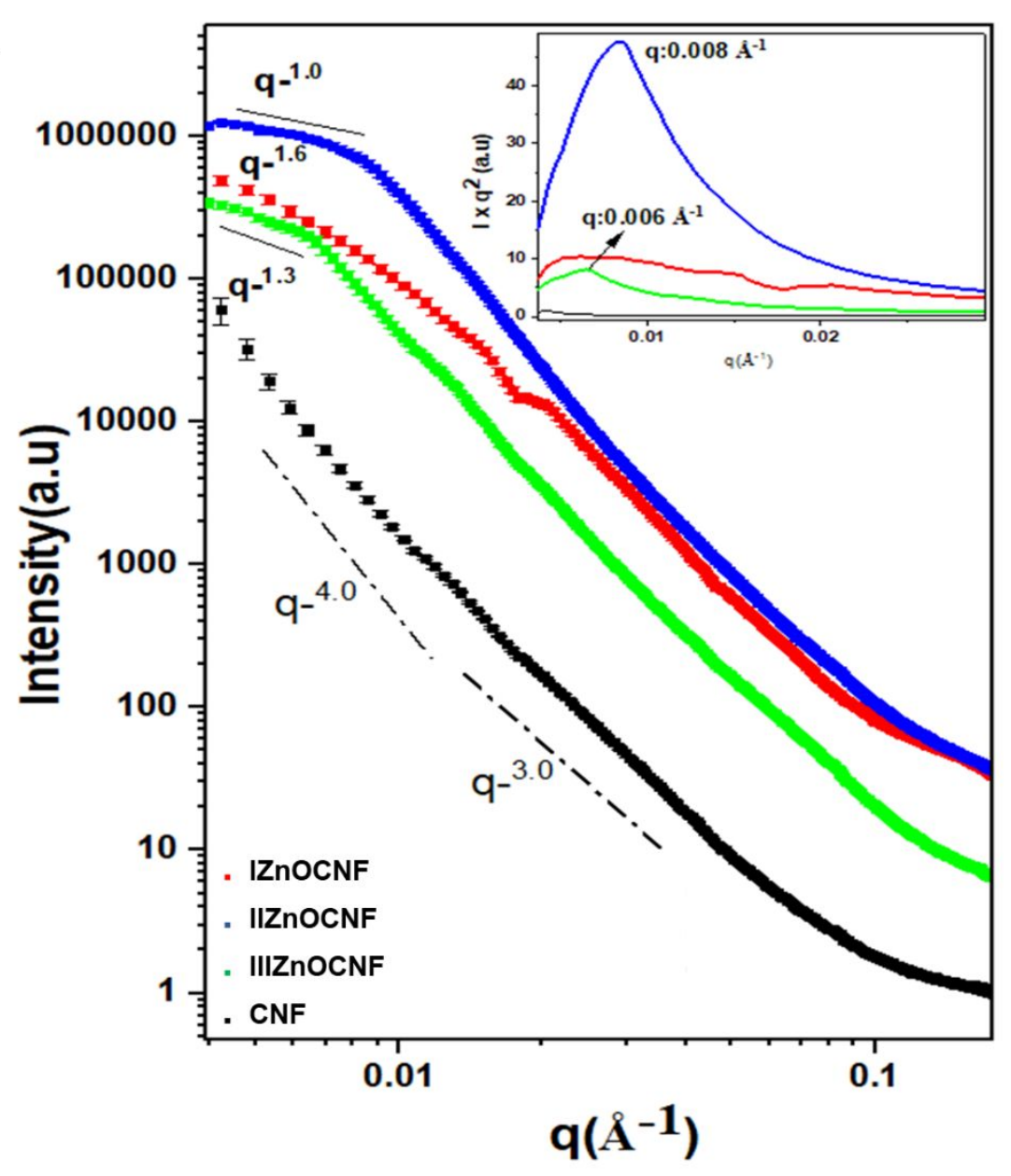

Fig. S3. a) SAXs scattering images, b) SAXS scattering curves of the pure CNF and three samples IZnOCNF, IIZnOCNF and IIIZnOCNF. Kratky plot inset. 


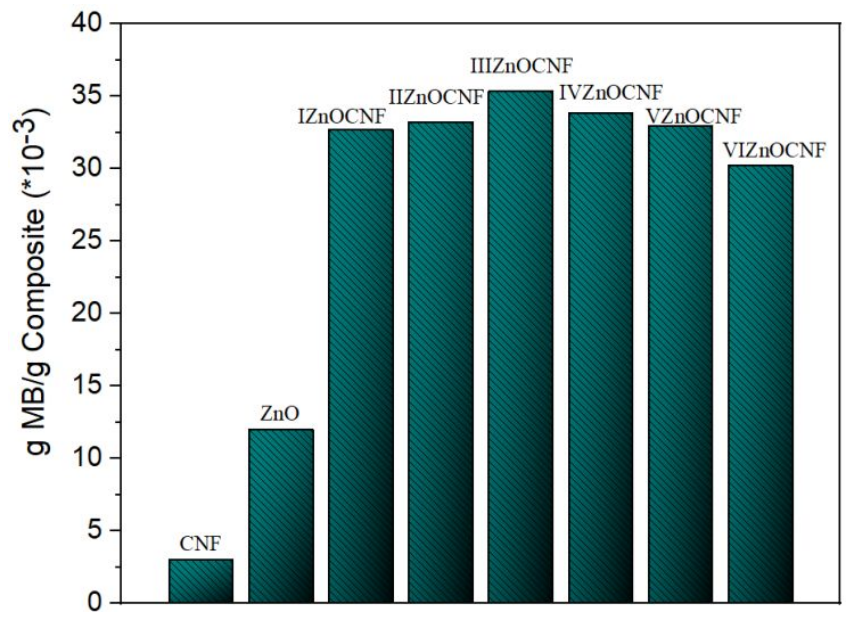

Fig. S4. The MB dye adsorption capacity of $0.2 \mathrm{~g}$ of each in-situ prepared composite after 60 minutes mixing under dark environment condition (5ppm MB and $100 \mathrm{~mL}$ solution).

a)

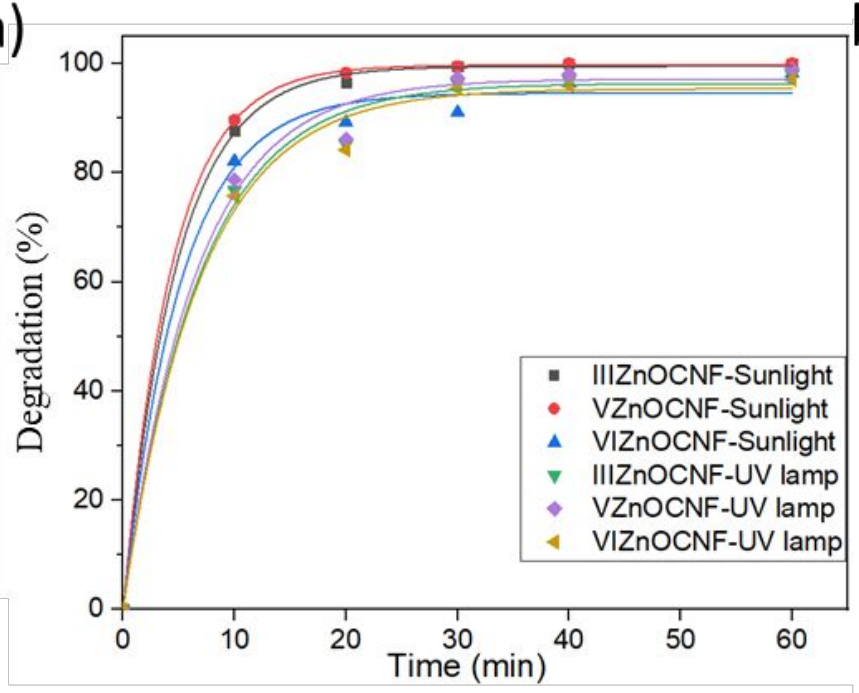

b)

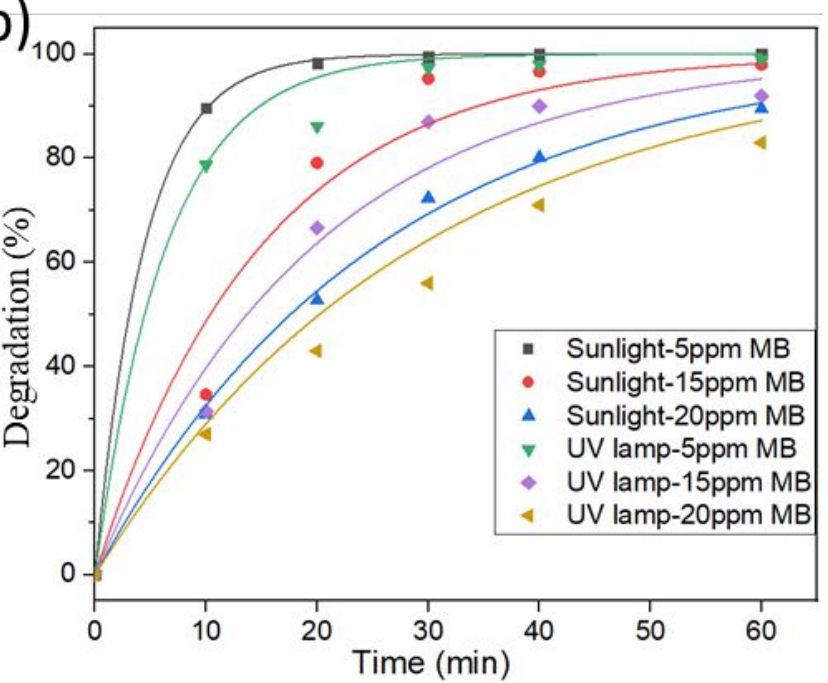

Figure S5. a) Degradation of MB dye by $0.2 \mathrm{~g}$ catalyst/100 $\mathrm{mL}$ water for IVZnOCNF,

VZnOCNF, VIZnOCNF composites under sunlight and UV lamp b) Degradation of 5, 15, and $20 \mathrm{ppm}$ concentrations of MB dye using $0.2 \mathrm{~g}$ VZnOCNF composite/100 mL water under sunlight and UV lamp. 
Table S2. Pseudo first order kinetic rate constants (k).

\begin{tabular}{|c|c|c|c|}
\hline Data-set & $\mathrm{k}$ value $\left(\mathrm{min}^{-1}\right)$ & Standard error $( \pm)$ & R-square \\
\hline IZnOCNF-sunlight & 0.023 & 0.002 & 0.992 \\
\hline IZnOCNF-UV light & 0.020 & 0.001 & 0.994 \\
\hline IIZnOCNF-sunlight & 0.079 & 0.001 & 1 \\
\hline IIZnOCNF- UV light & 0.073 & 0.011 & 0.978 \\
\hline IIIZnOCNF-sunlight & 0.183 & 0.02 & 0.999 \\
\hline IIIZnOCNF- UV light & 0.126 & 0.019 & 0.993 \\
\hline IVZnOCNF-sunlight & 0.180 & 0.02 & 0.999 \\
\hline IVZnOCNF- UV light & 0.131 & 0.017 & 0.992 \\
\hline VZnOCNF-sunlight & 0.225 & 0.003 & 0.999 \\
\hline VZnOCNF- UV light & 0.174 & 0.009 & 0.997 \\
\hline VIZnOCNF-sunlight & 0.165 & 0.024 & 0.982 \\
\hline VIZnOCNF- UV light & 0.125 & 0.009 & 0.971 \\
\hline
\end{tabular}

Table S3. Comparison of synthesized $\mathrm{ZnO} / \mathrm{CNF}$ composite with other metal oxide composite photocatalysts in the literature.

\begin{tabular}{|c|c|c|c|c|}
\hline M-O $_{x}$ or composite & Irradiation source & $\begin{array}{l}\text { MB Degradation under } \\
\text { irradiation (\%) }\end{array}$ & MB concentration & Ref. \\
\hline $\mathrm{ZnO}$ & $\begin{array}{c}4 \text { UV lamp-9 W-365 nm, } 1400-2000 \mu \mathrm{W} / \mathrm{cm}^{-1} \text { of } \\
\text { UVA, UVB }\end{array}$ & $\begin{array}{l}\text { After } 60 \mathrm{~min}: \\
82\end{array}$ & $10 \mathrm{ppm}$ & This study \\
\hline $\mathrm{ZnO}$ & $\begin{array}{c}\text { Sunlight, UV index } \approx 3-5,1500-2900 \mu \mathrm{W} / \mathrm{cm}^{-1} \text { of } \\
\text { UVA, UVB }\end{array}$ & $\begin{array}{l}\text { After } 60 \mathrm{~min}: \\
\quad 38\end{array}$ & $10 \mathrm{ppm}$ & This study \\
\hline $\mathrm{ZnO}$ & Four $4 \mathrm{~W}$ UV lamps & $\begin{array}{l}\text { After } 120 \mathrm{~min}: \\
\qquad 60\end{array}$ & $50 \mathrm{ppm}$ & 1 \\
\hline VZnOCNF & $\begin{array}{c}4 \text { UV lamp-9 W-365 nm, } 1400-2000 \mu \mathrm{W} / \mathrm{cm}^{-1} \text { of } \\
\text { UVA, UVB }\end{array}$ & $\begin{array}{l}\text { After } 60 \mathrm{~min}: \\
94\end{array}$ & $10 \mathrm{ppm}$ & This study \\
\hline VZnOCNF & $\begin{array}{l}\mathrm{UV} \text { index } \approx 3-5,1500-2900 \mu \mathrm{W} / \mathrm{cm}^{-1} \text { of UVA, } \\
\mathrm{UVB}\end{array}$ & $\begin{array}{l}\text { After } 10 \text { min: } \\
\quad>99\end{array}$ & $10 \mathrm{ppm}$ & This study \\
\hline $\mathrm{ZnO} /$ graphene & Visible light (metal halogen lamp, $\lambda \geq 420 \mathrm{~nm}$ ) & $\begin{array}{l}\text { After } 90 \text { min: } \\
\quad>99\end{array}$ & $20 \mathrm{ppm}$ & 2 \\
\hline
\end{tabular}




\begin{tabular}{|c|c|c|c|c|}
\hline $\mathrm{ZnS}-\mathrm{ZnO} /$ graphene & Visible light irradiation (400 W) & $\begin{array}{l}\text { After } 100 \mathrm{~min}: \\
\quad>99\end{array}$ & $10 \mathrm{ppm}$ & 3 \\
\hline $\mathrm{ZnO} @ \mathrm{C}-\mathrm{Ag}$ & AM $1.5100 \mathrm{~mW} / \mathrm{cm}^{2}$ simulated sunlight & $\begin{array}{l}\text { After } 20 \mathrm{~min}: \\
98\end{array}$ & $10 \mathrm{ppm}$ & 4 \\
\hline $\begin{array}{l}\mathrm{ZnO} / \mathrm{ZnFe}_{2} \mathrm{O}_{4} \text { Janus } \\
\text { Hollow Nanofibers }\end{array}$ & $150 \mathrm{~W}$ xenon lamp $\left(\sim 393 \mathrm{~mW} / \mathrm{cm}^{2}\right)$ & $\begin{array}{l}\text { After } 180 \mathrm{~min}: \\
\qquad 98\end{array}$ & $10 \mathrm{ppm}$ & 5 \\
\hline $\mathrm{SrTiO}_{3}$ & $\begin{array}{l}\text { High-pressure } 250 \mathrm{~W} \mathrm{Hg} \text { vapor lamp } \\
\text { Wavelength: } 410-700 \mathrm{~nm}\end{array}$ & After 120 min: 35 & $10 \mathrm{ppm}$ & 6 \\
\hline Porous graphene- $\mathrm{SrTiO}_{3}$ & $\begin{array}{c}\text { High-pressure } 250 \mathrm{~W} \mathrm{Hg} \text { vapor lamp } \\
\text { Wavelength: } 410-700 \mathrm{~nm}\end{array}$ & $\begin{array}{l}\text { After } 120 \mathrm{~min}: \\
\qquad 95\end{array}$ & $10 \mathrm{ppm}$ & 6 \\
\hline $\begin{array}{c}\text { Walnut-shaped } \\
\text { hierarchical } \\
\text { multihollow CdS } \\
\text { microspheres/ } \\
\text { coordination polymer }\end{array}$ & $8 \mathrm{~W}$ UV lamp Wavelength: $365 \mathrm{~nm}$ & $\begin{array}{l}\text { After } 60 \mathrm{~min} \text { : } \\
\qquad 88\end{array}$ & $1.5 \mathrm{ppm}$ & 7 \\
\hline $\mathrm{Cu}_{3} \mathrm{~N}$ & $\begin{array}{l}\text { KRATOS, universal arc lamp supply: } 250 \mathrm{~W} \text {, } \\
\text { 150XE }\end{array}$ & $\begin{array}{c}\text { After } 25 \text { min: } \\
\quad \sim 30\end{array}$ & Unknown & 8 \\
\hline $\begin{array}{c}\mathrm{Cu}_{3} \mathrm{~N} \text { Nanocrystals } \\
\text { Decorated with } \mathrm{Au} \\
\text { Nanoparticles }\end{array}$ & $\begin{array}{c}\text { KRATOS, universal arc lamp supply: } 250 \mathrm{~W} \text {, } \\
150 \mathrm{XE}\end{array}$ & $\begin{array}{l}\text { After } 25 \text { min: } \\
\qquad 95\end{array}$ & Unknown & 8 \\
\hline $\begin{array}{l}\text { Wrinkled Ultrathin } \\
\text { Graphitic } \mathrm{C}_{3} \mathrm{~N}_{4} \\
\text { Nanosheets }\end{array}$ & 300 W Xe lamp Wavelength: $420 \mathrm{~nm}$ & $\begin{array}{l}\text { After } 90 \mathrm{~min}: \\
\qquad 99\end{array}$ & $100 \mathrm{ppm}$ & 9 \\
\hline $\mathrm{TiO}_{2}$ & UV Hg-lamp (Heraeus TQ-150, $150 \mathrm{~W}$ ) & $\begin{array}{l}\text { After } 120 \mathrm{~min} \text { : } \\
\quad 80-100\end{array}$ & $10 \mathrm{ppm}$ & 10 \\
\hline $\mathrm{TiO}_{2}$ & $\begin{array}{l}\text { Between } 11 \text { am to } 2 \mathrm{pm} \text { under sunlight and the } \\
\text { solar intensity of } 1250 \times 100 \mathrm{Lu} \pm 100\end{array}$ & $\begin{array}{l}\text { After } 120 \text { min: } \\
\qquad 35\end{array}$ & $10 \mathrm{ppm}$ & 11 \\
\hline $\begin{array}{l}\mathrm{TiO}_{2} / \text { cashew nutshell } \\
\text { activated carbon } \\
\text { (CNSAC) }\end{array}$ & $\begin{array}{l}\text { Between } 11 \text { am to } 2 \mathrm{pm} \text { under sunlight and the } \\
\text { solar intensity of } 1250 \times 100 \mathrm{Lu} \pm 100\end{array}$ & $\begin{array}{l}\text { After } 120 \mathrm{~min}: \\
\qquad 94\end{array}$ & $10 \mathrm{ppm}$ & 11 \\
\hline
\end{tabular}




\begin{tabular}{|c|c|c|c|c|}
\hline $\begin{array}{c}\mathrm{TiO}_{2} \text { Nanofilms on } \\
\text { Polymeric Substrates }\end{array}$ & $9 \mathrm{~W}$ UVP compact UV lamp at $368 \mathrm{~nm}$ & $\begin{array}{c}\text { After } 120 \mathrm{~min}: \\
95\end{array}$ & $4 \mathrm{ppm}$ & 12 \\
\hline $\mathrm{Fe}_{2} \mathrm{O}_{3}$ & Osram, UltraVitalux E27, 300W & $\begin{array}{l}\text { After } 60 \mathrm{~min} \text { : } \\
\qquad 60-80\end{array}$ & $10 \mathrm{ppm}$ & 13 \\
\hline $\mathrm{Fe}_{2} \mathrm{O}_{3} / \mathrm{TiO}_{2}$ & $\begin{array}{c}\text { Visible } \\
\text { Xe-lamp (Hamamatsu L2274, } 150 \mathrm{~W} \text { ) }\end{array}$ & $\begin{array}{c}\text { After } 60 \mathrm{~min}: \\
93\end{array}$ & $10 \mathrm{ppm}$ & 13 \\
\hline $\mathrm{Nb}_{2} \mathrm{O}_{5}$ & $400 \mathrm{~W}$ mercury vapour lamp & $\begin{array}{c}\text { After } 180 \mathrm{~min} \text { : } \\
25-30\end{array}$ & $10 \mathrm{ppm}$ & 14 \\
\hline $\begin{array}{l}\text { Amorphous niobium } \\
\text { oxide hydrates shells }\end{array}$ & $\begin{array}{c}\text { visible light: } \\
\text { A } 300 \mathrm{~W} \text { xenon lamp }\end{array}$ & $\begin{array}{l}\text { After } 180 \mathrm{~min} \text { : } \\
72\end{array}$ & $10 \mathrm{ppm}$ & 15 \\
\hline
\end{tabular}

\section{References}

1. Chakrabarti, S.; Dutta, B. K., Photocatalytic degradation of model textile dyes in wastewater using ZnO as semiconductor catalyst. J Hazard Mater 2004, 112 (3), 269-278.

2. Ahmad, M.; Ahmed, E.; Hong, Z.; Xu, J.; Khalid, N.; Elhissi, A.; Ahmed, W., A facile one-step approach to synthesizing $\mathrm{ZnO} /$ graphene composites for enhanced degradation of methylene blue under visible light. Appl Surf Sci 2013, 274, 273-281.

3. Lonkar, S. P.; Pillai, V. V.; Alhassan, S. M., Facile and scalable production of heterostructured $\mathrm{ZnS}-\mathrm{ZnO} / \mathrm{Graphene}$ nano-photocatalysts for environmental remediation. Scientific reports $\mathbf{2 0 1 8 , 8}$ (1), 1-14.

4. $\quad$ Shen, Z.; Liang, P.; Wang, S.; Liu, L.; Liu, S., Green synthesis of carbon-and silver-modified hierarchical $\mathrm{ZnO}$ with excellent solar light driven photocatalytic performance. ACS Sustainable Chem Eng

2015, 3 (5), 1010-1016.

5. Liu, H.; Han, C.; Shao, C.; Yang, S.; Li, X.; Li, B.; Li, X.; Ma, J.; Liu, Y., ZnO/ZnFe $\mathrm{Z}_{4}$ Janus Hollow Nanofibers with Magnetic Separability for Photocatalytic Degradation of Water-Soluble Organic Dyes. ACS Appl Nano Mater 2019, 2 (8), 4879-4890.

6. Bantawal, H.; Sethi, M.; Shenoy, U. S.; Bhat, D. K., Porous Graphene Wrapped $\mathrm{SrTiO}_{3}$ Nanocomposite: $\mathrm{Sr}-\mathrm{C}$ Bond as an Effective Coadjutant for High Performance Photocatalytic Degradation of Methylene Blue. ACS Appl Nano Mater 2019, 2 (10), 6629-6636.

7. Thakur, S.; Das, P.; Mandal, S. K., Solvent-Induced Diversification of CdS Nanostructures for Photocatalytic Degradation of Methylene Blue. ACS Appl Nano Mater 2020.

8. Barman, D.; Paul, S.; Ghosh, S.; De, S. K., $\mathrm{Cu}_{3} \mathrm{~N}$ Nanocrystals Decorated with Au Nanoparticles for Photocatalytic Degradation of Organic Dyes. ACS Appl Nano Mater 2019, 2 (8), 5009-5019.

9. $\quad$ Gao, M.; Feng, J.; Zhang, Z.; Gu, M.; Wang, J.; Zeng, W.; Lv, Y.; Ren, Y.; Wei, T.; Fan, Z., Wrinkled Ultrathin Graphitic $\mathrm{C}_{3} \mathrm{~N}_{4}$ Nanosheets for Photocatalytic Degradation of Organic Wastewater. ACS Appl Nano Mater 2018, 1 (12), 6733-6741. 
10. Jallouli, N.; Elghniji, K.; Trabelsi, H.; Ksibi, M., Photocatalytic degradation of paracetamol on $\mathrm{TiO}_{2}$ nanoparticles and $\mathrm{TiO}_{2}$ /cellulosic fiber under UV and sunlight irradiation. Arab J Chem 2017, 10, S3640-S3645.

11. Ragupathy, S.; Raghu, K.; Prabu, P., Synthesis and characterization of $\mathrm{TiO}_{2}$ loaded cashew nut shell activated carbon and photocatalytic activity on $B G$ and $M B$ dyes under sunlight radiation. Spectrochim Acta A 2015, 138, 314-320.

12. Maleki, $\mathrm{H}$.; Bertola, $\mathrm{V}$., $\mathrm{TiO}_{2}$ Nanofilms on Polymeric Substrates for the Photocatalytic Degradation of Methylene Blue. ACS Appl Nano Mater 2019, 2 (11), 7237-7244.

13. Li, R.; Jia, Y.; Bu, N.; Wu, J.; Zhen, Q., Photocatalytic degradation of methyl blue using $\mathrm{Fe}_{2} \mathrm{O}_{3} / \mathrm{TiO}_{2}$ composite ceramics. J Alloys Compd 2015, 643, 88-93.

14. Kulkarni, A. K.; Praveen, C.; Sethi, Y. A.; Panmand, R. P.; Arbuj, S. S.; Naik, S. D.; Ghule, A. V.; Kale, B. B., Nanostructured N-doped orthorhombic $\mathrm{Nb}_{2} \mathrm{O}_{5}$ as an efficient stable photocatalyst for hydrogen generation under visible light. Dalton Trans 2017, 46 (43), 14859-14868.

15. Oliveira, L. C.; Oliveira, H. S.; Mayrink, G.; Mansur, H. S.; Mansur, A. A.; Moreira, R. L., Onepot synthesis of CdS@ $\mathrm{Nb}_{2} \mathrm{O}_{5}$ core-shell nanostructures with enhanced photocatalytic activity. Appl Catal, B 2014, 152, 403-412. 